\title{
HER2 status in breast cancer-an example of pharmacogenetic testing
}

\author{
Mark Kroese ${ }^{1} \quad$ Ron L Zimmern ${ }^{2} \quad$ Sarah E Pinder $^{3}$
}

$J$ R Soc Med 2007; 100:326-329

\section{SUMMARY}

The development of new drugs and associated pharmacogenetic tests will provide an increasing number of challenges to health care systems. In particular, how to evaluate their benefits, prioritize for commissioning purposes and implement a service to provide them in a timely manner. This paper presents an overview of HER2 testing for trastuzumab (Herceptin) treatment in breast cancer cases. Immunohistochemistry and fluorescence in situ hybridization laboratory techniques are described and their HER2 testing performances are compared. Future options for the national provision of HER2 testing by the National Health Service in the UK are also discussed.

\section{INTRODUCTION}

The recent controversy concerning the provision of trastuzumab (Herceptin) treatment in the UK has highlighted the difficulties of managing the introduction of new treatments and technologies in a resource-limited health care setting. ${ }^{1,2}$ Trastuzumab treatment requires HER2 testing to establish whether an individual is suitable for treatment. This is an example of a pharmacogenetic test.

Pharmacogenomics is the study of how genomic variation influences inter-individual variability in drug response. Pharmacogenetic tests identify the presence or absence of a particular gene variant which can influence an individual's response to a specific drug. It is necessary for commissioners of health care services and public health specialists to be aware of the key features of such new diagnostics.

\section{METHODS}

We conducted a MEDLINE/PubMed search up to December 2005, with search terms 'HER2', 'trastuzumab', 'FISH' and 'test'. References cited in published papers were reviewed to ensure that relevant articles were not being missed from electronic searches. In addition, one of the

${ }^{1}$ Consultant in Public Health Medicine and ${ }^{2}$ Director, Public Health Genetics Unit, Strangeways Research Laboratory, Worts Causeway, Cambridge CB1 8RN, UK; ${ }^{3}$ Consultant Breast Pathologist, Histopathology Department, Addenbrooke's NHS Trust, UK authors has specialist expertise in HER2 testing and her experience informed this review.

\section{HER2 RECEPTOR}

The HER2 gene is a member of the type 1 tyrosine kinase growth factor receptor family that is found on the long arm of chromosome 17. The biology of the HER2 receptor is complex, but it is involved in both cell differentiation and proliferation. HER2 protein is over-expressed in 25-30\% of human breast cancers; in $90-95 \%$ of these cases overexpression is a direct result of gene amplification.

In gene amplification, the normal DNA replication process is seriously flawed. The result is that instead of making a single copy of a region of a chromosome, many copies are produced. This leads to the production of many copies of the genes that are located on that region of the chromosome.

HER2 protein over-expression (i.e. a greater number of $H E R 2$ receptors than normal) correlates with poor clinical prognosis. ${ }^{3}$ It is associated with high grade tumours, lymph node involvement, greater risk of recurrence and relative resistance to some types of chemotherapy. ${ }^{4}$ This results in shorter disease-free survival and overall survival from breast cancer. ${ }^{5}$

Trastuzumab is a recombinant humanized monoclonal antibody that specifically targets the HER2 receptor. There is increasing evidence of the clinical benefits of trastuzumab treatment in cases of invasive breast cancer in which HER2 has been shown to be over-expressed or is amplified. ${ }^{6-9}$ It does not provide any benefit to those cases of breast cancer with normal expression levels of HER2. It is important to note that it is not a question of the presence or absence of $H E R 2$, but of the over-expression or amplification of HER2 that is of interest. HER2 test-positive status is defined as the over-expression or amplification of HER2, and this is a prerequisite for the use of trastuzumab. This emphasizes the importance of accurate HER2 testing and targeting of treatment to the patients who are most likely to benefit, because trastuzumab is associated with cardiotoxicity and is expensive. The estimated cost of treatment for one year with trastuzumab in the UK is in the range of $£ 20000$ $£ 30$ 000. Patients with a history of cardiac problems such as myocardial infarction or poorly controlled hypertension are at greater risk of cardiotoxicity, as are individuals who have previously received chemotherapy with anthracyclines. It is 
important that cardiac function is assessed before and during treatment to prevent cardiotoxicity.

At present, HER2 testing is carried out principally by two methods in the UK: immunohistochemistry (IHC) and fluorescence in situ hybridization (FISH). IHC identifies HER2 receptor over-expression and FISH identifies HER2 gene amplification. These tests are carried out on tumour tissue samples, which are fixed in buffered formalin and embedded in paraffin wax.

\section{IMMUNOHISTOCHEMISTRY}

IHC is a technique that uses antibodies as a tool to detect protein expression. Monoclonal or polyclonal antibodies complementary to the antigen of interest are labelled with a marker (either visible by light microscopy or fluorescence), allowing detection of the antibodies bound to regions of protein expression in a tissue sample. Diagnostic immunohistochemistry is widely used, for example, to detect tissue markers associated with specific cancers.

\section{FLUORESCENCE IN SITU HYBRIDIZATION}

FISH is a technique used to identify the presence of specific chromosomes or chromosomal regions through hybridization (attachment) of fluorescently labelled DNA probes to denatured chromosomal DNA. Examination under fluorescent lighting detects the presence of the hybridized fluorescent signal (and hence presence of the chromosome material).

\section{HER2 TESTING IN THE UK}

Current UK recommendations for HER2 testing have been developed as referenced in the National Institute of Excellence (NICE) Technology Appraisal Guidance 34, Guidance on the Use of Trastuzumab for the Treatment of Advanced Breast Cancer. A two-phase testing process is recommended, involving both IHC and FISH; IHC is the most frequently used initial technique in the UK. No other techniques are supported for determining HER2 status at this time. The UK testing algorithm, which is similar to other national testing algorithms, ${ }^{10}$ is shown in Figure 1.

For IHC testing, standardized commercial assay kits are available, such as Herceptest (DakoCytomation). In-house assays can also be used, but present evidence is that these fare less well in the UK National External Quality Assessment Scheme for Immunocytochemistry (UK NEQAS ICC). For IHC HER2 testing, only membrane staining of the invasive tumour should be considered when scoring IHC tests. The scoring method is shown in Table 1. Scoring is subjective, requires training and expertise and scorer bias has been documented. One of the main concerns with IHC is that there is evidence of significant inter-observer variation in the assessment of staining, which can lead to misclassification of HER2 status as confirmed by FISH HER2

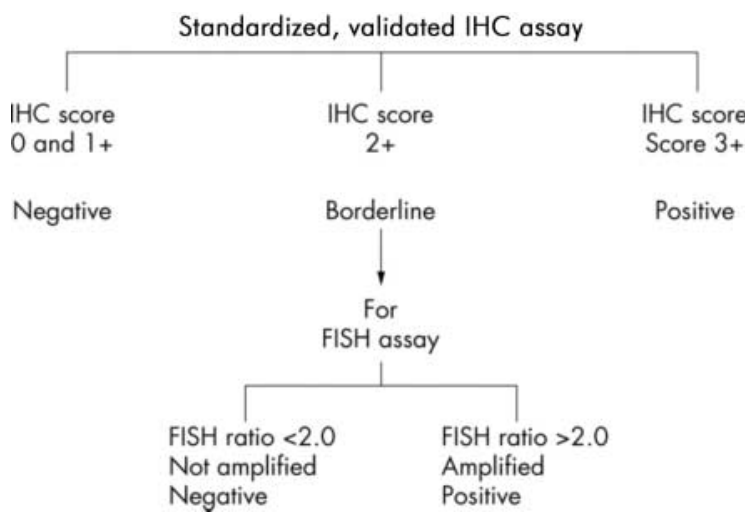

Figure 1 UK recommended testing algorithm ${ }^{15}$

testing. ${ }^{11-13}$ In practice, those cases scored as $2+$ with IHC for HER2 are considered not as positives but as borderline, requiring further assessment with FISH HER2 testing.

All clinical laboratories performing IHC HER2 testing as predictive or prognostic tests must participate in an external quality assurance (EQA) programme such as UK NEQAS ICC. Laboratories providing IHC HER2 testing should carry out a minimum of 250 assays each year. This standard is supported by evidence that laboratories undertaking a low number of tests generally perform poorly. ${ }^{14}$

The results of FISH HER2 tests are conventionally expressed as the ratio of HER2 signal to the chromosome 17 control signal. Tumours showing a ratio of more than two are considered positive. It is recommended that clinical laboratories providing FISH HER2 testing should carry out a minimum of 100 tests per year in order to maintain standards of testing. A smaller minimum number has been recommended for FISH testing than for IHC testing because FISH is accepted to be a more discriminatory test, has greater ease of methodological standardization and less observer variation. ${ }^{15}$ Clinical laboratories providing FISH HER2 testing are expected to join the EQA scheme coordinated by NEQAS, at present running in pilot form.

Table 1 Recommended immunohistochemistry scoring method ${ }^{15}$

\begin{tabular}{lll}
$\begin{array}{l}\text { Score } \\
\text { to } \\
\text { report } \\
\text { overexpression } \\
\text { assessment }\end{array}$ & Staining pattern \\
\hline 0 & Negative & $\begin{array}{l}\text { No staining is seen, or membrane } \\
\text { staining in less than } 10 \% \text { of tumour cells } \\
\text { A faint/barely perceptible membrane } \\
\text { staining is detected in more than } 10 \% \text { of } \\
\text { tumour cells. The cells are only stained } \\
\text { in part of the membrane } \\
\text { Weak to moderate complete membrane } \\
\text { staining is seen in more than } 10 \% \text { of } \\
\text { tumour cells }\end{array}$ \\
& Negative & $\begin{array}{l}\text { Strong complete membrane staining is } \\
\text { seen in more than } 10 \% \text { of tumour cells }\end{array}$ \\
$3+$ & Positive &
\end{tabular}




\section{COMPARISON OF IHC AND FISH TESTING FOR HER2}

FISH has been shown to be more accurate because of the stability of the DNA target; it is also easier to interpret and the inter-observer variation is lower because FISH is a quantitative rather than qualitative test. ${ }^{11}$ The better performance of FISH HER2 testing has meant that it is now used as the reference standard for HER2 tests. It is therefore not surprising that evidence from the UK and internationally shows that IHC performs poorly in comparison and has higher false-negative and false-positive rates.

In addition to being more accurate in establishing HER2 status, FISH has also been shown to be a better predictor than IHC of response to trastuzumab treatment and overall prognosis. ${ }^{5,16}$ It should be noted that many of these studies are not comparable; FISH is invariably performed in large centres with established expertise whilst IHC is routinely available in most histopathology laboratories where only small numbers of tests may be performed. In addition, there is a tendency in some series to classify IHC $2+$ scores as positive and then to report those cases without gene amplification by FISH as false negatives.

IHC testing is cheaper and quicker than the FISH method. It is also a technique that is well-established and routinely performed - for markers other than HER2 - in most histopathology laboratories. The FISH technique is labour-intensive, more expensive and requires a significant capital outlay for equipment, as FISH is not part of routine histopathological practice. ${ }^{17}$ Developing a FISH testing service for HER2 would also require considerable training for laboratory personnel. One UK regional histopathology laboratory has estimated the cost of an IHC HER2 kit at $£ 30$ and for a FISH HER2 kit at $£ 70$. These estimates are for tests only and do not include the overall cost of providing a testing service, such as personnel or other resources. Automated high-throughput technologies for FISH testing are also being developed.

The relatively poorer performance of IHC HER2 testing is partly due to inter-observer variation associated with the subjective grading of IHC test results, and this is particularly marked for the $2+$ group. This is illustrated in the test performance data of IHC compared to FISH HER2 testing from two UK studies ${ }^{16,18}$ presented in Tables 2 and 3. The study by Dowsett et al. was larger $(n=426$, Study One) compared to that carried out by Ellis et al. $(n=114$, Study Two).

Using the published data from the study by Dowsett et al. ${ }^{18}$ it is possible to calculate the sensitivity, specificity, positive predictive value and negative predictive value of IHC HER2 testing against the reference standard of FISH HER2 testing. The IHC test performance results (as per UK specificity $98 \%$, positive predictive value $94 \%$ and negative predictive value $99 \%$. These results are similar to that obtained from a US study. ${ }^{17}$

HER2 testing using either IHC or FISH requires specialist histopathology expertise. Tissue samples need to be assessed and the invasive part of the tumour has to be distinguished from the non-invasive and normal areas. Only the invasive tumour section of the sample should be tested, and in situ carcinoma not included in the assessment, otherwise invalid results will be obtained. This raises the problem of providing a FISH testing service where there is limited access to Consultant Histopathology expertise.

Early evidence of clinical effectiveness supports the developing consensus that there should be primary FISH testing for HER2. ${ }^{11,12,15,19}$ However, there is insufficient health economic data on the cost effectiveness of the different HER2 testing methods and strategies. One study from the US found that it was more cost effective to carry out primary FISH HER2 testing or FISH HER2 confirmation testing of all IHC HER2 test positive samples compared to IHC HER2 testing alone or FISH HER2 testing of weakly IHC HER2 positive samples. ${ }^{20}$ Such a study investigating the cost-effectiveness of the different testing strategies in the UK is urgently needed.

\section{CONCLUSION}

Current UK guidelines for HER2 testing appear to provide acceptable test performance. Such performance is dependant

Table 2 Proportion of sample tested by IHC HER2 testing score

\begin{tabular}{lll}
\hline IHC score & \multicolumn{2}{c}{ Proportion of study sample tested } \\
\cline { 2 - 3 } & Study one & Study two \\
\hline $0 / 1+$ & $63 \%$ & $63 \%$ \\
$2+$ & $13 \%$ & $19 \%$ \\
$3+$ & $24 \%$ & $18 \%$ \\
\hline
\end{tabular}

Table 3 FISH HER2 testing status by IHC HER2 testing score

\begin{tabular}{|c|c|c|c|c|}
\hline \multirow{3}{*}{$\begin{array}{l}\text { IHC } \\
\text { score }\end{array}$} & \multicolumn{4}{|c|}{ FISH results } \\
\hline & \multicolumn{2}{|l|}{ Positive } & \multicolumn{2}{|l|}{ Negative } \\
\hline & Study one & Study two & Study one & Study two \\
\hline $0 / 1+$ & $0.7 \%$ & $7 \%$ & $99.3 \%$ & $93 \%$ \\
\hline $2+$ & $48 \%$ & $33 \%$ & $52 \%$ & $67 \%$ \\
\hline $3+$ & $94 \%$ & $90 \%$ & $6 \%$ & $10 \%$ \\
\hline
\end{tabular}

It is important to note that the studies used different FISH ratio cut-offs. Study One used $\geqslant 2.0$ and Study Two used $\geqslant 2.2$. 
on rigorous external quality assurance schemes and training. The false positive rate (as defined by FISH) of $5-10 \%$ for IHC HER2 testing ( $3+$ group) is of concern and the clinical significance of such results should be investigated; it is at present not clear whether and to what extent these patients will respond to trastuzumab.

An alternative approach is to provide primary FISH HER2 testing. There is evidence supporting primary FISH HER2 testing but cost-effectiveness data is still awaited. If the NHS does proceed to primary FISH HER2 testing, this would require an investment of resources and a significant period of time to establish capacity. In the interim, a mixed approach of IHC and FISH HER2 testing similar to the current UK HER2 testing guidelines would still be needed whilst laboratories are equipped and trained to carry out FISH testing. However, a strategic decision is needed on whether to proceed to primary FISH HER2 testing with a staged approach to implementation or to continue with current guidelines.

Whichever HER2 testing strategy is chosen, it will be necessary for commissioning groups and cancer networks to identify which laboratories will provide HER2 testing. Each will be expected to develop and maintain expertise in this area, carry out the minimum recommended number of tests as per UK guidelines, participate in the appropriate EQA programme and have rigorous and robust quality control systems. There is evidence from a recent survey by the charity CancerBACUP that indicates HER2 test provision is variable in the UK at present. ${ }^{21}$

Further research is urgently required to confirm what the cut-off ratio should be for the definition of 'amplification' versus 'normal' levels as identified by FISH HER2 testing and its impact in terms of outcomes with trastuzumab treatment.

The benefits of introducing FISH molecular diagnostics into general pathology laboratory services will not be limited to HER2 testing. It is expected that this development will allow laboratories to provide a wider range of services in the future as more pharmacogenetic tests similar to HER2 become available. HER-2 testing is the first of a number of molecular targets which will further influence the choice of targeted drug treatment in cancer and others will also require assessment by IHC and/or FISH to detect protein and gene abnormalities respectively. FISH testing is already a wellestablished technique in cytogenetic laboratories and there is an opportunity for an interdisciplinary approach to the development of this type of diagnostic service.

\section{Competing interests None declared.}

Funding None.

\section{REFERENCES}

1 Dent R, Clemons M. Adjuvant trastuzumab for breast cancer. BMJ 2005;331:1035-6

2 Herceptin and early breast cancer: a moment for caution. Lancet 2005;366: 1673

3 Ross JS, Fletcher JA, Linette GP, et al. The Her-2/neu gene and protein in breast cancer 2003: biomarker and target of therapy. Oncologist 2003;8:307-25

4 Burstein HJ. The distinctive nature of HER2 - positive breast cancers. $N$ Engl J Med 2005;353:1652-4

5 Pauletti G, Dandekar S, Rong H, et al. Assessment of methods for tissue-based detection of the HER-2/neu alteration in human breast cancer: a direct comparison of fluorescence in situ hybridization and immunohistochemistry. J Clin Oncol 2000;18:3651-64

6 Hortobagyi GN. Trastuzumab in the treatment of breast cancer. N Engl J Med 2005;353:1734-6

7 Piccart-Gebhart MJ, Procter M, Leyland-Jones B, et al. Trastuzumab after adjuvant chemotherapy in HER2-positive breast cancer. $N$ Engl J Med 2005;353:1659-72

8 NICE. Guidance on the Use of Trastuzumab for the Treatment of Advanced Breast Cancer. Technological Appraisal Guidance, No. 34. London: NICE, 2002: 1-14

9 Romond EH, Perez EA, Bryant J, et al. Trastuzumab plus adjuvant chemotherapy for operable HER2-positive breast cancer. $N$ Engl $J$ Med 2005;353:1673-84

10 Bilous M, Dowsett M, Hanna W, et al. Current perspectives on HER2 testing: a review of national testing guidelines. Mod Pathol 2003; 16:173-82

11 Bartlett JM, Going JJ, Mallon EA, et al. Evaluating HER2 amplification and over-expression in breast cancer. J Pathol 2001;195:422-8

12 Bartlett J, Mallon E, Cooke T. The clinical evaluation of HER-2 status: which test to use? J Pathol 2003;199:411-7

13 Rhodes A, Jasani B, Anderson E, Dodson AR, Balaton AJ. Evaluation of HER-2/neu immunohistochemical assay sensitivity and scoring on formalin-fixed and paraffin-processed cell lines and breast tumors: a comparative study involving results from laboratories in 21 countries. Am J Clin Pathol 2002;118:408-17

14 Paik S, Bryant J, Tan-Chiu E, et al. Real-world performance of HER2 testing-National Surgical Adjuvant Breast and Bowel Project experience. J Natl Cancer Inst 2002;94:852-4

15 Ellis IO, Bartlett J, Dowsett M, et al. Best Practice No 176: Updated recommendations for HER2 testing in the UK. J Clin Pathol 2004;57:233-7

16 Ellis CM, Dyson MJ, Stephenson TJ, Maltby EL. HER2 amplification status in breast cancer: a comparison between immunohistochemical staining and fluorescence in situ hybridization using manual and automated quantitative image analysis scoring techniques. J Clin Pathol 2005;58:710-4

17 Yaziji H, Goldstein LC, Barry TS, et al. HER-2 Testing in Breast Cancer Using Parallel Tissue-Based Methods. JAMA 2004;291: 1972-7

18 Dowsett M, Bartlett J, Ellis IO, et al. Correlation between immunohistochemistry (HercepTest) and fluorescence in situ hybridization (FISH) for HER-2 in 426 breast carcinomas from 37 centres. J Pathol 2003;199:418-23

19 Lewis F, Jackson P, Lane S, Coast G, Hanby AM. Testing for HER2 in breast cancer. Histopathology 2004;45:207-17

20 Elkin EB, Weinstein MC, Winer EP, Kuntz KM, Schnitt SJ, Weeks JC. HER-2 testing and trastuzumab therapy for metastatic breast cancer: a cost-effectiveness analysis. J Clin Oncol 2004;22:854-63

21 CancerBACUP Cancer Drug Testing Shortfall. BBC News, 5 Oct 2005. Available at http://news.bbc.co.uk/1/hi/health/4312476.stm (accessed 05/03/2007). 